$$
\begin{aligned}
\text { Hence } \mathrm{N}_{\mathrm{n}} & =2 \mathrm{~N}_{\mathrm{n}-1}+1 ; \\
\therefore \mathrm{N}_{\mathrm{n}}+1 & =2\left\{\mathrm{~N}_{\mathrm{n}-1}+1\right\}=2\left\{2\left(\mathrm{~N}_{\mathrm{n}-2}+1\right)\right\} \\
& =\ldots \ldots \ldots \ldots \ldots \ldots \\
& =2^{\mathrm{n}-1}\left(\mathrm{~N}_{1}+1\right)=2^{\mathrm{n}-1}(1+1) \\
& =2^{\mathrm{n}} ; \\
\therefore \mathrm{N}_{\mathrm{n}} & =2^{\mathrm{n}}-1 .
\end{aligned}
$$

(2.) A little inspection will show that any disc has to be moved twice as often as the one immediately greater; and since the $n^{\text {th }}$ disc is moved only once, it follows that

$$
\begin{aligned}
\mathrm{N}_{\mathrm{n}} & =1+2+2^{2}+\ldots \ldots+2^{\mathrm{n}-1} \\
& =\frac{2^{\mathrm{n}}-1}{2-1}=2^{\mathrm{n}}-1 .
\end{aligned}
$$

§ 3.-To accomplish the actual moves a simple rule may be given, as follows:-

To shift an even number of discs from $A$ to $C$, move, by one step at a time, the odd numbers round $A B C$, counter-clockwise, the even numbers round $\mathrm{ACB}$, clockwise.

To shift an odd number of discs the directions are reversed.

\title{
Note on Spherical Trigonometry.
}

By R. E. Allardice, M.A.

In the first volume of Gergonne's Annales de Mathématiques (1810-11), there is a paper by Lhuilier, in which he gives properties of the right-angled spherical triangle, analogous to the following properties of the right-angled plane triangle :

1. The square on the hypotenuse is equal to the sum of the squares on the other two sides;

2. If a perpendicular be drawn from the right angle to the hypotenuse, the square on each side is equal to the rectangle contained by the hypotenuse and the adjacent segment of the hypotenuse;

3. The squares on the sides are to one another as the adjacent segments of the hypotenuse ;

4. The square on the perpendicular is equal to the rectangle contained by the segments of the hypotenuse;

5. The hypotenuse, the sides, and the perpendicular are in proportion. 
Now the spherical triangle that has one angle equal to the sum of the other two, is in some respects analogous to the right-angled plane triangle; and it is one of the infinite number of spherical triangles which become right-angled when the radius of the sphere is made infinite. It is, for example, the triangle about which a semicircle may be described; and it is the triangle of maximum area, when two sides are given.

The following is a table containing the properties of the right. angled spherical triangle, given by Lhuilier, and the properties of the triangle referred to above, amalogous in each case to the five properties of the right-angled plane triangle already given.

Denote the hypotenuse by $c$, the other sides by $a$ and $b$, the perpendicular by $h$, and the segments of the base by $l$ and $m$.

\section{$\mathrm{O}$ a right angle.}

1. $\operatorname{Sin}^{2} \frac{1}{2} c=\sin ^{2} \frac{1}{2} b \cos ^{2} \frac{1}{2} a+\sin ^{2} \frac{1}{2} a \cos ^{2} \frac{1}{2} b$.

2. $\sin ^{2} b: \sin c . \sin m=1: \cos l$; $\operatorname{Sin}^{2} a: \sin c \cdot \sin l=1: \cos m$.

3. $\operatorname{Sin}^{2} a: \sin ^{2} b=\sin 2 l: \sin 2 m$.

4. $\operatorname{Sin}^{2} h: \sin l \sin m=1: \cos l \cos m$.

5. Sin $c: \sin a=\sin b: \sin h$.

$$
\mathbf{C}=\mathbf{A}+\mathbf{B} \text {. }
$$

1. $\operatorname{Sin}^{2} \frac{1}{2} c=\sin ^{2} \frac{1}{2} a+\sin ^{2} \frac{1}{2} b$.

2. $\operatorname{Tan} b \tan \frac{1}{2} b=\tan m \tan \frac{1}{2} c$. Tan $a \tan \frac{1}{2} a=\tan l \tan \frac{1}{2} c$.

3. $\sin ^{2} \frac{1}{2} a: \sin ^{2} \frac{1}{2} b=\sin l: \sin m$.

4. $\operatorname{Tan}^{2} \frac{1}{2} h=\tan \frac{1}{2} l \tan \frac{1}{2} m$.

5. $\operatorname{Sin} \frac{1}{2} c: \sin \frac{1}{2} a=\sin \frac{1}{2} b: \frac{1}{2} \sin h$.

In both cases the above formule give the properties of the right-angled plane triangle, when the radius of the sphere is made infinite, the sine and the tangent of any angle then becoming equal to the angle, and the cosine of any angle unity. Formulæe may also be established for this triangle, analogous to those for the rightangled triangle, connecting any three of the elements of the triangle. 